\title{
Comparison of trunk muscle thickness according to the type of feedback during spinal stabilization exercise in standing posture
}

\author{
Hee-Ji Lee ${ }^{a(i)}$,Su-Ha Lee ${ }^{a(i)}$, Seong-Joo Lee ${ }^{b(i)}$, Chang-Hyung Lee ${ }^{c(i)}$, Dae-Sung Park ${ }^{d(1)}$ \\ a'Department of Physical Therapy, The Graduate School, Konyang University, Daejeon, Republic of Korea \\ ${ }^{\mathrm{b}}$ Department of Rehabilitation Medicine, Seoul National University Bundang Hospital, Seongnam, Republic of Korea \\ ${ }^{\mathrm{c}}$ Rehabilitation Medicine, Pusan National University School of Medicine, Research Institute for the Convergence of Biomedical Science and \\ Technology, Pusan National University Yangsan Hospital, Yangsan, Republic of Korea \\ ${ }^{d}$ Department of Physical Therapy, College of Medical Science, Konyang University, Daejeon, Republic of Korea
}

Objective: Patients with low back pain can possibly have impaired core muscle function, which is the common cause of low back pain. Spinal stabilization exercises are recommended for prevention and reinforcement. This study aimed to compare the effects of different types of feedback on abdominal and lumbar multifidus (LM) muscle recruitment during spinal stabilization exercises.

Design: Cross-sectional study.

Methods: Fifty-seven healthy subjects ( $\mathrm{sex}=$ male $21 /$ female 36 , age $=21.28 \pm 1.60$ years) were divided into three different groups: the control group $(n=19)$, the auditory feedback (AF) group $(n=19)$, and the visual and auditory feedback (VAF) group ( $n=19)$. The control group received no feedback, whereas the AF group only received AF during exercises and the VAF group received the AF and visual feedback through the real-time ultrasound images. The main outcome measure was the assessment of the thickness of the abdominal muscles and LM measured by a dual ultrasound.

Results: When VAF was applied, the thickness of the transverse abdominis significantly increased rather than when feedback was not applied or with AF only $(p<0.05)$. The VAF group showed significant differences in both the control group and the AF group in the post-hoc test $(p<0.05)$, and there was no significant difference between the control group and the AF group.

Conclusions: With spinal stabilization exercises, VAF should be applied in standing posture for healthy adults to further promote the production of effective contractions.

Key Words: Abdominal muscles, Audio feedback, Standing position, Ultrasonography, Visual feedback

\section{Introduction}

Core muscles are divided into global and local muscles [1]. The external oblique (EO) and internal oblique (IO), which are included in the global muscles, produce torque and transfer the load directly between the thoracic cage and the pelvis [2]. Local muscles stabilize the lumbar region, such as the transverse abdominis (TrA) and lumbar multifidus (LM). Particularly, LM controls the spinal segment's neutral zone in harmony with the deep abdominal muscles
[3], whereas the TrA supports the spine and controls the pressure of the abdominal cavity [4]. To maintain proper spinal stability to prevent back pain it is important to contract these two types of muscles simultaneously [5].

It is possible for patients with low back pain to have impaired core muscle function, which is the common cause of low back pain [6]. The use of spinal stabilization exercises, which can maintain the balance between the deep abdominal muscles and the trunk extensors by acting like a corset of the trunk, is one method to prevent the occurrence and re-

Received: 31 August, 2020 Revised: 18 September, 2020 Accepted: 18 September, 2020

Corresponding author: Dae-Sung Park (ORCID https://orcid.org/0000-0003-4258-0878)

Department of Physical Therapy, College of Medical Science, Konyang University, 158 Gwanjeodong-ro, Seo-gu, Daejeon 35365, Republic of Korea Tel: 82-42-600-6419 Fax: 82-42-600-6565 E-mail: daeric@konyang.ac.kr

(c) This is an Open-Access article distributed under the terms of the Creative Commons Attribution Non-Commercial License (http://creativecommons.org/licenses/ by-nc/4.0) which permits unrestricted non-commercial use, distribution, and reproduction in any medium, provided the original work is properly cited.

Copyright $@ 2020$ Korean Academy of Physical Therapy Rehabilitation Science 
currence the back pain [7].

One common exercise for spinal stabilization is the abdominal drawing-in maneuver (ADIM) that is used for strengthening and educating spinal muscles [8]. This maneuver is created to provide the co-activation of the $\operatorname{Tr} A$ and LM to stabilize the trunk [9]. Previous studies show that spinal stabilization exercises are carried out in supine, prone, 4-point kneeling, and sitting posture [10,11]. Training in these postures is effective in patients with acute back pain. However, training in standing posture is the most effective for patients with chronic back pain or healthy adults [12,13]. Hence, few studies have been conducted in standing posture.

Ultrasound is a highly reliable equipment used to assess whether the muscles are contracting well [14]. Also, it is non-invasive, easy to apply, economical, and can visualize the contraction of the core muscles to provide an immediate visual feedback effect [15]. Therefore, it is especially used as a means of feedback to aid exercise. For those who contract the spinal muscles for the first time or have weakened spinal muscles and has the advantage of improving motor control or motor performance, the real-time ultrasound image feedback provides corrective education [16,17]. In addition, auditory feedback (AF) is an effective external feedback technique that is applied by someone else to the subject through verbal instructions or praise and can enhance the ability to perform the exercise [18]. According to prior studies, subjects prefer the application of AF to the application of visual feedback during exercise [19]. As a way of accurate exercise learning, the use of ultrasound biofeedback reduced the number of trials required to perform correct abdominal hollowing rather than the application of verbal feedback [20].

The aim of this study was to investigate the effects of visual and auditory feedback (VAF) or AF on abdominal and LM muscle thickness, as well as to determine if the application of real-time ultrasound image and $\mathrm{AF}$ together will increase the muscle thickness the most effectively.

\section{Methods}

\section{Participants}

Fifty-seven healthy subjects (sex=male 21 /female 36 ), comprised mainly of students from Konyang University in Daejeon, South Korea, who received sufficient explanation about the purpose and methods of this study, were recruited. Exclusion criteria included history of surgery of spine or upper body, current musculoskeletal or neurological disorders affecting upper or lower extremities, back pain within the last 6 months, past spinal fracture, spinal deformity, history of skeletal or neuromuscular disease, a skin disease, and sensitive skin that is difficult to measure by ultrasound. After assigning members as 12 male and 7 female to each group, the fifty-seven subjects were randomly assigned within each gender and equally into three groups: the control group, $\mathrm{AF}$ group, and the VAF group. With no significant differences between the groups, the mean age, height, and weight of the subjects in each group were as follows: in the control group, the mean values of the study subject characteristics were $21.26 \pm 1.69,165.10 \pm 7.02 \mathrm{~cm}$, and $61.32 \pm 9.91 \mathrm{~kg}$, respectively; in the AF group, $21.53 \pm 1.71$ years, $166.68 \pm$ $10.52 \mathrm{~cm}, 62.03 \pm 13.83 \mathrm{~kg}$, respectively; and in the VAF group, the mean values were $21.05 \pm 1.43$ years, $165.53 \pm 8.85$ $\mathrm{cm}, 60.17 \pm 10.75 \mathrm{~kg}$, respectively. The study was approved by the Institutional review board of Konyang University (IRB No. KYU-2019-248-02). All participants signed the consent in advance, and the rights of participants were protected.

\section{Equipment}

\section{Ultrasound}

A belt with a 5-8 MHZ dual linear transducer (LV8-5N60A2; TELEMED, Vilnius, Lithyania) was used to measure the thickness of the abdominal and LM muscles and provide real-time ultrasound image feedback (Figure 1). By passive adjustment, each transducer could be moved from side to side. Each of the transducer's head was secured in a high-density supporting foam block on both sides to minimize movement between the transducer and the abdomen.

\section{Resistance pole}

The resistance pole (Core Stix Fitness System; Core Stix, Los Angeles, CA, USA) is an exercise device that can apply many functional multi-joint exercises to everyone and can

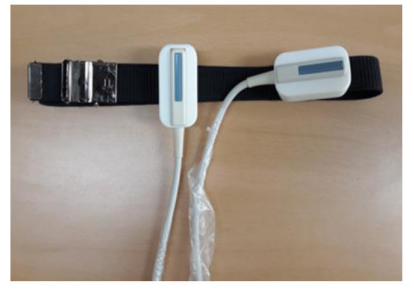

A

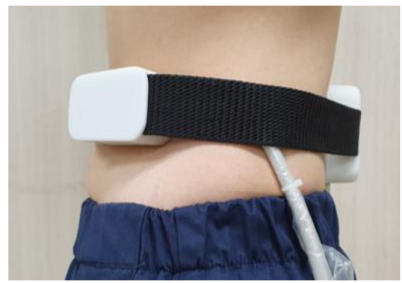

B
Figure 1. (A) A belt with a dual ultrasound transducer. (B) Wearing a belt. 
effectively motivate spinal muscle activity even in standing posture. According to the degree of resistance, the sticks are classified using colors. The result of the preliminary test shows that the white stick led both men and women to exercise in a correct posture. Hence, the experiment was conducted by unifying it with a white stick (Figure 2).

\section{Measurement with ultrasound}

The images of the muscles were measured using a wearable belt. Wearing a belt and placing each transducer in the proper position were performed in a comfortable standing posture to visualize the abdominal muscles and the LM.

The transducer head location was marked at a point $2.5 \mathrm{~cm}$ anterior to the midpoint between the 12 th rib and the superior border of the ilium passing through the left mid-axillary line to measure the left abdominal muscles. The transducer was placed at that point transversely [21]. The L4 spinous process was marked with a marker to measure the left LM, and the transducer was placed longitudinally $2 \mathrm{~cm}$ away from the marking to the left. The transducer was adjusted until the L4-5 zygapophyseal joint was identified at the center of the screen at this point [22].

At the end of exhalation, ultrasound measurements were conducted three times in a comfortable, resting standing posture and were conducted three times in each of the three different exercise postures. A total of 12 images were obtained for each subject. Subjects took a 30 -second break after every measurement. The target was measured after maintaining the end posture of the exercise when measuring in an exercise posture for 3 seconds and it was measured three times for each posture.

Using the RadiAnt DICOM Viewer 4.6.9 software (Medixant, Poznań, Poland), the muscle thickness of the ac-

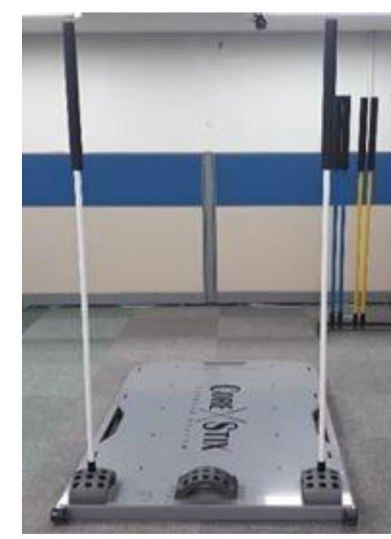

Figure 2. Resistance pole. quired image was measured. The muscle thickness of the EO, IO, and TrA was measured vertically at a point $13 \mathrm{~mm}$ away from the endpoint of the TrA [23], and the thickness of the LM was measured along the line from the L4-5 zygapophyseal joint to the superior border of the LM (Figure 3) [24]. The measured value was the average value of the three measurements.

\section{Procedures}

The subjects were pre-trained about the ADIM before the exercises and were performed in a crook-lying posture on a simple bed. The subjects exercised using the resistance pole while wearing a wearable belt with ultrasonic transducers. All the exercises proceeded with two white sticks, which were fixated on each side vertically. The starting posture of all exercises were to stand with both feet shoulder-width apart and to hold the sticks with the elbow joints flexed to $90^{\circ}$. The first exercise was the 'reverse fly (RF)'. Maintaining the starting posture, the participants gathered and maintained the sticks on both sides in parallel. The second exercise was the 'push'. Maintaining the starting posture, the participants pushed the sticks on both sides forward in parallel. At this time, they pushed until the elbows were fully extended to $180^{\circ}$ while keeping the body in a straight line. The third exercise was the 'pull'. Maintaining the starting posture, the participants pulled and maintained the sticks on both sides in parallel. At this time, the examiner put her hand on the subjects' back, and the subjects were to pull the sticks until their arms reached the examiner's arm keeping their body in a straight line. By applying one of the following three different feedback methods, the exercises were con-

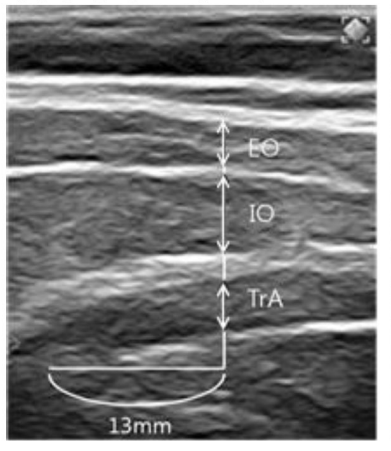

A

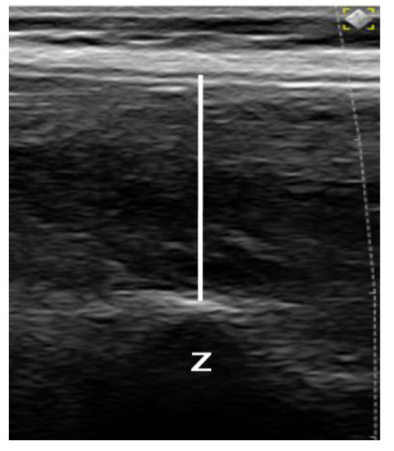

B
Figure 3. (A) Thickness measurements of the abdominal muscles. (B) Thickness measurements of the lumbar multifidus. EO: external oblique, IO: internal oblique, TrA: transverse abdominis, Z: zygapophyseal joint. 
ducted together to the assigned group: The control group did not receive any feedback during exercises; the AF group only received AF such as 'do the exercise by pulling the belly' and 'maintain the power on the lower abdomen while breathing'; the VAF group received visual feedback through the real-time ultrasound images to view for the changes in the thickness of their abdominal muscles and LM in addition to the AF.

\section{Statistical analysis}

The Shapiro-Wilk test was conducted as a normality test. One-way analysis of variance was used to compare the thickness of the EO, IO, TrA, and LM, depending on the type of feedback. The Tukey test was used as a post hoc test. For data management and statistical analysis, IBM SPSS Statistics for Windows, Version 20.0 (IBM Co., Armonk, NY, USA) was used, and the statistical significance level was set to 0.05 .

\section{Results}

\section{Characteristics of subjects}

Table 1 shows the general characteristics of the subjects. During the experiment period, none of the 57 participants who were recruited had dropped out. Hence, the number of final participants in the study was equal to the initial number of subjects, which was 57 .

\section{Comparison of the thickness of abdominal muscles and LM according to the methods of feedback}

The thickness of the TrA, EO, IO, and LM in each posture of the exercises is shown in Table 2 between each group. There was a significant increase in TrA thickness in the condition with VAF compared with the control or AF in all three different postures of exercise (RF: $6.35 \pm 1.62 \mathrm{~mm}$ vs. $5.17 \pm$ $0.91 \mathrm{~mm}$ or $5.26 \pm 1.00 \mathrm{~mm}, p=0.007$; pull: $6.63 \pm 1.52 \mathrm{~mm}$ vs.
$5.52 \pm 0.99 \mathrm{~mm}$ or $5.46 \pm 1.17 \mathrm{~mm}, p=0.008$; push: $6.55 \pm 1.52$ $\mathrm{mm}$ vs. $5.34 \pm 1.22 \mathrm{~mm}$ or $5.44 \pm 1.17 \mathrm{~mm}, p=0.010)$. The VAF group showed significant differences in both the control group and the AF group in a post hoc test $(p<0.05)$, and there was no significant difference between the control group and the AF group. The thickness of the EO, IO, and LM had no significant difference between the types of feedback.

\section{Discussion}

This study was conducted to investigate what the effects on spinal muscle contractions during spinal stabilization exercise performed by healthy adults in standing posture were in two cases; when only AF was applied and when only the VAF was applied.

The result showed that the thickness of the TrA in the group that applied VAF had increased significantly than the group that did not apply any feedback and in the group that applied only AF: RF, $p<0.007$; pull, $p<0.008$; push, $p<0.010$. When doing the ADIM in the crook-lying position, one study also showed a significant increase in the thickness of the TrA in a group that applied visual feedback rather than in a group that did not apply feedback [16]. The difference in thickness of the TrA before and after the exercise was $1.19 \pm 0.37 \mathrm{~mm}$ [16], but in this study, there was a much bigger difference: $2.55 \pm 0.36 \mathrm{~mm}$ at RF, $2.83 \pm 0.26 \mathrm{~mm}$ at pull, and $2.75 \pm 0.26 \mathrm{~mm}$ at push. When doing the ADIM, the thickness of the TrA increased significantly in the standing posture than in the crook-lying posture [25]. As such, this study was conducted in standing posture, and there was also $\mathrm{AF}$ and stick resistance, and therefore it is believed that there was a greater increase in the thickness of the TrA than other studies that conducted ADIM in the crook-lying posture. Hence, it will be necessary to conduct spinal stabilization exercises while applying VAF in standing posture to pro-

Table 1. General characteristics of subjects

$(\mathrm{N}=57)$

\begin{tabular}{lcccc}
\hline \multicolumn{1}{c}{ Characteristic } & Control $(\mathrm{n}=19)$ & AF group $(\mathrm{n}=19)$ & VAF group $(\mathrm{n}=19)$ & $\mathrm{F} / \mathrm{Z}(p)$ \\
\hline Sex $($ female $/$ male $)$ & $12 / 7$ & $12 / 7$ & $12 / 7$ & $<0.000(1.000)$ \\
Age $(\mathrm{y})$ & $21.26(1.69)$ & $21.53(1.71)$ & $21.05(1.43)$ & $0.409(0.666) / 21.28(1.60)$ \\
Height $(\mathrm{cm})$ & $165.10(7.02)$ & $166.68(10.52)$ & $165.53(8.85)$ & $0.160(0.853) / 165.77(8.78)$ \\
Weight $(\mathrm{kg})$ & $61.32(9.91)$ & $62.03(13.83)$ & $60.17(10.75)$ & $0.124(0.884) / 61.17(11.43)$ \\
BMI $\left(\mathrm{kg} / \mathrm{m}^{2}\right)$ & $22.50(1.45)$ & $22.33(1.66)$ & $21.96(0.95)$ & $0.222(0.802) / 22.26(1.33)$ \\
\hline
\end{tabular}

Values are presented as number only or mean (SD).

Control: control group, AF: auditory feedback, VAF: visual and auditory feedback, BMI: body mass index. 
Table 2. Comparison of the thickness of abdominal muscles and LM according to the methods of feedback during core stabilization exercise in standing posture (unit: $\mathrm{mm}$ )

\begin{tabular}{|c|c|c|c|c|}
\hline Characteristic & Control $(n=19)$ & AF group $(n=19)$ & VAF group $(n=19)$ & $\mathrm{F}(p)$ \\
\hline \multicolumn{5}{|l|}{$\operatorname{TrA}$} \\
\hline Resting & $3.93(1.05)$ & $4.09(0.93)$ & $3.8(1.26)$ & $0.341(0.713)$ \\
\hline $\mathrm{RF}$ & $5.17(0.91)$ & $5.26(1.00)$ & $6.35(1.62)^{\mathrm{ab}}$ & $5.481(0.007)$ \\
\hline Pull & $5.52(0.99)$ & $5.46(1.17)$ & $6.63(1.52)^{\mathrm{ab}}$ & $5.360(0.008)$ \\
\hline Push & $5.34(1.22)$ & $5.44(1.17)$ & $6.55(1.52)^{\mathrm{ab}}$ & $4.988(0.010)$ \\
\hline $\mathrm{F}(p)$ & $0.517(0.599)$ & $0.190(0.828)$ & $0.174(0.841)$ & \\
\hline \multicolumn{5}{|l|}{$\mathrm{EO}$} \\
\hline Resting & $7.21(2.05)$ & $7.84(2.50)$ & $7.68(2.38)$ & $0.192(0.826)$ \\
\hline $\mathrm{RF}$ & $4.60(1.25)$ & $4.64(1.92)$ & $4.77(1.36)$ & $0.067(0.935)$ \\
\hline Pull & $4.13(1.39)$ & $4.56(1.91)$ & $4.40(1.27)$ & $0.370(0.693)$ \\
\hline Push & $4.60(1.31)$ & $4.80(2.02)$ & $4.66(1.18)$ & $0.085(0.918)$ \\
\hline $\mathrm{F}(p)$ & $0.781(0.463)$ & $0.074(0.928)$ & $0.439(0.647)$ & \\
\hline \multicolumn{5}{|l|}{ IO } \\
\hline Resting & $7.21(2.05)$ & $7.84(2.51)$ & $7.68(2.38)$ & $0.375(0.689)$ \\
\hline $\mathrm{RF}$ & $9.34(3.00)$ & $10.88(4.21)$ & $10.68(3.81)$ & $0.970(0.386)$ \\
\hline Pull & $10.37(3.17)$ & $11.33(3.88)$ & $10.98(4.16)$ & $0.312(0.734)$ \\
\hline Push & $9.66(3.29)$ & $10.66(4.00)$ & $10.98(3.60)$ & $0.683(0.510)$ \\
\hline $\mathrm{F}(p)$ & $0.537(0.588)$ & $0.136(0.873)$ & $0.038(0.963)$ & \\
\hline \multicolumn{5}{|l|}{ LM } \\
\hline Resting & $28.99(1.52)$ & $28.80(5.35)$ & $27.10(6.06)$ & $0.565(0.571)$ \\
\hline $\mathrm{RF}$ & $29.26(6.45)$ & $29.23(6.83)$ & $26.47(5.36)$ & $1.253(0.294)$ \\
\hline Pull & $29.46(7.08)$ & $29.25(6.30)$ & $26.94(4.75)$ & $0.992(0.377)$ \\
\hline Push & $31.36(6.55)$ & $31.00(7.27)$ & $28.02(5.71)$ & $10.491(0.234)$ \\
\hline $\mathrm{F}(p)$ & $0.571(0.568)$ & $0.418(0.661)$ & $0.431(0.652)$ & \\
\hline
\end{tabular}

Values are presented as mean (SD).

LM: lumbar multifidus, Control: control group, AF: auditory feedback, VAF: visual and auditory feedback, TrA: transverse abdominis, RF: reverse fly, EO: external oblique, IO: internal oblique.

${ }^{\mathrm{a}} \mathrm{S}$ Significant difference in comparison with control $(p<0.05)$, ${ }^{\mathrm{b}}$ Significant difference in comparison with $\mathrm{AF}(p<0.05)$.

duce more effective TrA contractions in healthy adults.

The thickness of the EO did not vary significantly in all three postures following the two types of feedback. Other prior studies comparing the pre-post muscle thickness in a group that applied real-time ultrasound image feedback with another group that did not apply any feedback in the supine posture did not show significant differences [26,27]. There was also no significant difference in the study that compared the muscle thickness before and after exercise of the two groups that apply AF or not in a 4-point kneeling posture [27]. As such, the change in thickness of the EO caused by the feedback showed similar results as previous studies.

The thickness of the IO did not vary significantly in all three postures depending on the two types of feedback. This is consistent with several prior studies [16,27], and because the amount of change in thickness are smaller in the standing posture than in supine during the ADIM, it is considered that it showed little difference [25].
The thickness of the LM also did not show significant differences in all types of feedback. When performing repetitive training through visual image feedback, one study measured the changes in muscle thickness, with a significant increase in muscle thickness [28]. While the study involved multiple repetitive training, this study performed only three measurements. Therefore, it is considered to show different results. In addition, another study that compared the thickness of the LM in the prone posture and the standing posture respectively showed significant changes in the prone posture, but little changes in the standing posture [29]. Because the LM is in a state of more contraction in the standing posture than in the lying or sitting postures, it is thought that there would have been no major changes in contraction [30].

Therefore, a single transducer is applied several times, or the two transducers are applied simultaneously, to compare the left and right thickness of the same muscles or the thickness of the two different muscles in the same exercise [31]. 
However, because the subject cannot perform a perfectly equal amount of exercise at every time, these methods may lead to inaccuracy of the results [32]. The dual transducer that was used in this study could measure an equal amount of exercise perfectly at every time with simultaneous measurement of the thickness of multiple muscles and also show the subjects two screens simultaneously in real-time and keep the transducers in the same position by the self-produced belt.

Applying AF by the side of the subject when he or she performs spinal stabilization exercises with visual feedback may lead to more effective contraction of the TrA than simply explaining how to perform or applying visual feedbacks without any verbal instructions in clinical settings based on the results of this study.

The additional limitations are as follows: First, subjects for this study were recruited in the limited area of healthy adults in their 20s and were measured only once, making it difficult to generalize the results of this study for all ages and other patients with chronic back pain, and it is difficult to know the effect of long-term training. Second, the resistance pole exercise was applied to both male and female with the white stick uniformly. They may have felt the degree of resistance differently depending on the individual differences. Therefore, future research would require the inclusions of people of various ages, patients with chronic back pain, or long-term intervention studies. It would also require applying colors to the sticks of the resistance pole separately according to the sex or degree of resistance of the subject.

In conclusion, the thickness of the $\operatorname{TrA}$ increased significantly when real-time ultrasound image feedback and AF were applied together than without feedback or with AF only based on the results of this study. The thickness of the EO, IO, and LM had no significant difference between the types of feedback. Hence, it is considered that VAF should be applied together with spinal stabilization exercises performed in a standing posture to healthy adults to produce more effective TrA muscle contractions.

\section{Conflict of Interest}

The authors declared no potential conflicts of interest with respect to the authorship and/or publication of this article.

\section{References}

1. Bergmark A. Stability of the lumbar spine. A study in mechanical engineering. Acta Orthop Scand Suppl 1989;230:1-54.

2. Imai A, Kaneoka K, Okubo Y, Shiina I, Tatsumura M, Izumi S, et al. Trunk muscle activity during lumbar stabilization exercises on both a stable and unstable surface. J Orthop Sports Phys Ther 2010;40:369-75.

3. O'Sullivan PB, Phyty GD, Twomey LT, Allison GT. Evaluation of specific stabilizing exercise in the treatment of chronic low back pain with radiologic diagnosis of spondylolysis or spondylolisthesis. Spine 1997;22:2959-67.

4. Misuri G, Colagrande S, Gorini M, Iandelli I, Mancini M, Duranti R, et al. In vivo ultrasound assessment of respiratory function of abdominal muscles in normal subjects. Eur Respir J 1997;10:2861-7.

5. McGill SM. Low back disorders: evidenced-based prevention and rehabilitation. 2nd ed. Champaign (IL): Human Kinetics; 2007.

6. Hides JA, Lambrecht G, Stanton WR, Damann V. Changes in multifidus and abdominal muscle size in response to microgravity: possible implications for low back pain research. Eur Spine J 2016;25 Suppl 1:175-82.

7. Hides JA, Jull GA, Richardson CA. Long-term effects of specific stabilizing exercises for first-episode low back pain. Spine 2001; 26:E243-8.

8. Ferreira ML, Ferreira PH, Latimer J, Herbert RD, Hodges PW, Jennings MD, et al. Comparison of general exercise, motor control exercise and spinal manipulative therapy for chronic low back pain: a randomized trial. Pain 2007;131:31-7.

9. eyhen DS, Miltenberger CE, Deiters HM, Del Toro YM, Pulliam JN, Childs JD, et al. The use of ultrasound imaging of the abdominal drawing-in maneuver in subjects with low back pain. J Orthop Sports Phys Ther 2005;35:346-55.

10. Akuthota V, Nadler SF. Core strengthening. Arch Phys Med Rehabil 2004;85(3 Suppl 1):S86-92.

11. Marshall PW, Murphy BA. Core stability exercises on and off a Swiss ball. Arch Phys Med Rehabil 2005;86:242-9.

12. Jung DE, Kim K, Lee SK. Comparison of muscle activities using a pressure biofeedback unit during abdominal muscle training performed by normal adults in the standing and supine positions. J Phys Ther Sci 2014;26:191-3.

13. Chan ST, Fung PK, Ng NY, Ngan TL, Chong MY, Tang CN, et al. Dynamic changes of elasticity, cross-sectional area, and fat infiltration of multifidus at different postures in men with chronic low back pain. Spine J 2012;12:381-8.

14. Taghipour M, Mohseni-Bandpei MA, Behtash H, Abdollahi I, Rajabzadeh F, Pourahmadi MR, et al. Reliability of real-time ultrasound imaging for the assessment of trunk stabilizer muscles: a systematic review of the literature. J Ultrasound Med 2019;38: $15-26$.

15. Thompson JA, O'Sullivan PB. Levator plate movement during voluntary pelvic floor muscle contraction in subjects with incontinence and prolapse: a cross-sectional study and review. Int Urogynecol J Pelvic Floor Dysfunct 2003;14:84-8.

16. Kwon NH, Lee HO, Park DJ. The use of real-time ultrasound imaging for feedback during abdominal hollowing. J Korean Soc 
Phys Med 2011;6:303-310.

17. Hides JA, Richardson CA, Jull GA. Use of real-time ultrasound imaging for feedback in rehabilitation. Man Ther 1998;3:12531.

18. Brody EB, Hatfield BD, Spalding TW, Frazer MB, Caherty FJ. The effect of a psyching strategy on neuromuscular activation and force production in strength-trained men. Res Q Exerc Sport 2000;71:162-70

19. Yoo H, Maeng W, Lee J. Desigining a feedback for exercises using a wearable device. J HCI Soc Korea 2016;11:23-30.

20. Henry SM, Westervelt KC. The use of real-time ultrasound feedback in teaching abdominal hollowing exercises to healthy subjects. J Orthop Sports Phys Ther 2005;35:338-45.

21. Ishida H, Suehiro T, Suzuki K, Yoneda T, Watanabe S. Influence of the ultrasound transducer tilt on muscle thickness and echo intensity of the rectus femoris muscle of healthy subjects. J Phys Ther Sci 2017;29:2190-3.

22. Larivière C, Gagnon D, De Oliveira E Jr, Henry SM, Mecheri H, Dumas JP. Ultrasound measures of the lumbar multifidus: effect of task and transducer position on reliability. PM R 2013;5:67887.

23. Cho M. The effects of bridge exercise with the abdominal drawing-in maneuver on an unstable surface on the abdominal muscle thickness of healthy adults. J Phys Ther Sci 2015;27:255-7.

24. Sions JM, Velasco TO, Teyhen DS, Hicks GE. Ultrasound imaging: intraexaminer and interexaminer reliability for multifidus muscle thickness assessment in adults aged 60 to 85 years versus younger adults. J Orthop Sports Phys Ther 2014;44:425-34.

25. Mew R. Comparison of changes in abdominal muscle thickness between standing and crook lying during active abdominal hol- lowing using ultrasound imaging. Man Ther 2009;14:690-5.

26. Lee DH, Hong SK, Lee YS, Kim CH, Hwang JM, Lee Z, et al. Is abdominal hollowing exercise using real-time ultrasound imaging feedback helpful for selective strengthening of the transversus abdominis muscle?: a prospective, randomized, parallel-group, comparative study. Medicine (Baltimore) 2018;97: e11369.

27. Critchley D. Instructing pelvic floor contraction facilitates transversus abdominis thickness increase during low-abdominal hollowing. Physiother Res Int 2002;7:65-75.

28. Van K, Hides JA, Richardson CA. The use of real-time ultrasound imaging for biofeedback of lumbar multifidus muscle contraction in healthy subjects. J Orthop Sports Phys Ther 2006;36: 920-5.

29. Sweeney N, O'Sullivan C, Kelly G. Multifidus muscle size and percentage thickness changes among patients with unilateral chronic low back pain (CLBP) and healthy controls in prone and standing. Man Ther 2014;19:433-9.

30. Lee SW, Chan CK, Lam TS, Lam C, Lau NC, Lau RW, et al. Relationship between low back pain and lumbar multifidus size at different postures. Spine 2006;31:2258-62.

31. Mannion AF, Pulkovski N, Gubler D, Gorelick M, O'Riordan D, Loupas T, et al. Muscle thickness changes during abdominal hollowing: an assessment of between-day measurement error in controls and patients with chronic low back pain. Eur Spine J 2008; 17:494-501.

32. Koppenhaver SL, Hebert JJ, Fritz JM, Parent EC, Teyhen DS, Magel JS. Reliability of rehabilitative ultrasound imaging of the transversus abdominis and lumbar multifidus muscles. Arch Phys Med Rehabil 2009;90:87-94. 